\title{
Failure of Friedel's Law in Electron Diffraction Scattering in Bilayer Graphene and Graphene with Defects
}

\author{
Hwang Su Kim
}

\author{
Department of Physics, Kyungsung University, Busan 608-736, Korea (jwa@ks.ac.kr)
}

Single layer graphene (SLG) or bilayer graphene (BLG) often has been considered as a two dimensional crystal objects. This treatment gives an implication for the electron scattering in SLG and BLG to be the kinematical. If this is valid, Friedel's law (FRL) must hold in experiments of the transmission electron microscopy of SLG or BLG materials: i.e. at the [0001] orientation, the inverse symmetry of electron diffraction amplitudes $\left|\phi_{\mathrm{g}}\right| \approx\left|\phi_{-\mathrm{g}}\right|$ should be observed. On the other hand, if SLG or BLG is assumed to be just the section of one or two atomic layers perpendicular to [0001] direction of a perfect graphite crystal, the dynamical theory with Bloch wave approach can be applied to the materials as shown in $[1,2]$. In contrary to the kinematical treatment, FRL does not hold in the dynamical diffraction especially for BLG as shown in Table 1. This result essentially comes from that graphite is non-centrosymmetric crystal with 3-fold rotational symmetry to the [0001] axis. It has long been known that FRL fails for non-centrosymmetric crystals in the electron dynamical diffraction [3]. In Table 1 the calculated dynamical diffraction intensity for SLG and BLG are listed. In this calculation the effect of up to $\pm 6^{\text {th }}$ higher order Laue zone reflections were taken into account. The each group of g-reflections such as $\{10.0\}_{\mathrm{A}}$ and $\{-10.0\}_{\mathrm{B}} /\{11.0\}_{\mathrm{A}}$ and $\{-1-$ $1.0\}_{\text {B }}$ represents 3-fold symmetry equivalent reflections with the inversion of $\mathbf{g}$ (subscription of A and B). In BLG the failure of FRL can be seen clearly for $\{10.0\}$ reflections. Even for SLG the small difference of intensity between $\{10.0\}_{\mathrm{A}}$ and $\{-10.0\}_{\mathrm{B}}$ reflections can be noted. If the higher Laue zone reflections are ignored, FRL holds because the projected potential along to [0001] direction is centrosymmetry. However in cases of SLG or BLG with defects, FRL is violated in the diffraction regardless the higher Laue zone reflections are ignored or not, because the resulting potential is non-centrosymmetry. Recently Lee et al. [4] have shown the diffraction pattern obtained at a $200 \mathrm{kV}$ TEM from a region containing several overlapped SLG and BLG or the folded sheets. In this paper we report an investigation for whether this diffraction pattern indeed shows the failure of FRL, as discussed above with the dynamical theory.

The diffraction pattern of Fig. 3(A) in [4] displays seven distinct diffraction sets with $\{10.0\}$ and $\{11.0\}$ reflections only with different orientations. Unfortunately some diffraction spots are missed by the beam stopper. Thus only the four patterns among the seven sets have 12 full reflections of $\{10.0\}$ and $\{11.0\}$. Table 2 is listed the measured intensity for each spots in this four patterns. In the listed intensity the diffuse background intensity of $\sim 45$ for the $\{10.0\}$ reflections was removed. This measurement has done with Scion Image software in the scale of $0 \sim 255$. The statistical uncertainty of the measurement of intensity is assumed to be $\sim \sqrt{I}_{\mathrm{g}}: \mathrm{I}_{\mathrm{g}}=\left|\phi_{\mathrm{g}}\right|^{2}$. For this assumption we followed a general Gaussian statistical rule of $\sim \sqrt{ } \mathrm{N}$ uncertainty in $\mathrm{N}$ measurements of events. Here we put the measured number $\mathrm{N}$ from the software as $I_{\mathrm{g}}$-intensity. If $\mathrm{I}_{\mathrm{g} 2}>\mathrm{I}_{\mathrm{g} 1}$ and $\Delta \mathrm{I}_{\mathrm{g}}=\mathrm{I}_{\mathrm{g} 2}-\mathrm{I}_{\mathrm{g} 1}>\sqrt{\mathrm{I}_{\mathrm{g} 2} \text {, }}$ then $\Delta \mathrm{I}_{\mathrm{g}}$ is considered to have statistically meaningful difference as $\mathrm{I}_{\mathrm{g} 2} \neq \mathrm{I}_{\mathrm{g} 1}$ in realty. Under this criterion one can see that the diffraction patterns listed in Table 2 definitely have not 3 -fold symmetry and many reflections loss the inverse symmetry of intensity which means the failure of FRL. For instance, in No. 1 pattern for (10.0) and (-10.0), $\Delta \mathrm{I}_{\mathrm{g}}=115-73(42)>\sqrt{ } 115(\sim 10.7)$ and for (11.0) and (-1-1.0), $\Delta \mathrm{I}_{\mathrm{g}}=62-27(35)>\sqrt{ } 62(\sim 7.9)$; in No. 2 pattern for (1-1.0) and $(-11.0), \Delta \mathrm{I}_{\mathrm{g}}=130$ $116(14)>\sqrt{ } 130(\sim 11.4)$ and for $(-1-1.0)$ and (11.0), $\Delta \mathrm{I}_{\mathrm{g}}=89-67(22)>\sqrt{89}(\sim 9.4)$; in No. 3 pattern for 
(01.0) and (0-1.0), $\Delta \mathrm{I}_{\mathrm{g}}=127-107(20)>\sqrt{ } 127(\sim 11.3)$ and for $(-21.0)$ and $(2-1.0), \Delta \mathrm{I}_{\mathrm{g}}=197-170$ $(27)>\sqrt{ } 197(\sim 14.0)$; in No. 4 pattern for $(0-1.0)$ and $(01.0), \Delta \mathrm{I}_{\mathrm{g}}=173-157(16)>\sqrt{ } 173(\sim 13.2)$ and for $(-21.0)$ and (2-1.0), $\Delta \mathrm{I}_{\mathrm{g}}=240-223(17)>\sqrt{ } 240(\sim 15.5)$. In the calculation work it was checked the symmetry breaking due to specimen tilting up to $\sim 11^{\circ}$. Because the specimen is ultra thin this effect turned out to be really small and can be ignored as not for this case. In the measured intensity the significant violation of 3-fold symmetry is an indication that the specimen area taken the diffraction was overall non-centrosymmetry in atomic positions. This aspect seems to be due to defects such as the overlapping of SLG and BLG or the folded sheets and/or an irregular slight stretch. It should be noted that even for this case the kinematical treatment of scattering is expected to generally supports FRL for such as low angle scattering reflections of $\{10.0\}$ and $\{11.0\}$. Interestingly in Fig. 3(A) in [4] the violation of FRL can be seen very clearly in the diffuse diffraction intensities around the central spot. Here it must be noted that the failure of FRL generally more prevails in the lower angle scattering such as $\{10.0\}$ reflections and the diffuse scattering around the central spot: this fact is thought to be due to the effect of the dynamical scattering indeed. The numerical calculations using the dynamical theory have given satisfactory explanation for the observation of the symmetry breaking including the violation of FRL discussed so far. In conclusion even in case SLG or BLG specimen like a two dimensional crystal objects the dynamical diffraction theory including the higher order Laue zone reflections must be employed for correct analysis for electron diffraction microscopy images.

[1] H.S. Kim, Microc. Microanal. 16(Suppl 2) (2010) 274-275.

[2] H.S. Kim, Korean J. Microscopy 40 (2010) 21-28.

[3] R. Serneels et al. phys. stat. sol. (b) 58 (1973) 277-292,.

[4] Z.H. Lee et al., Microc. Microanal. 15(Suppl 2) (2009) 124-125.

\begin{tabular}{|c|c|c|c|c|}
\hline \multirow{2}{*}{ Group } & \multirow{2}{*}{$\mathrm{h}$} & \multirow{2}{*}{$\mathrm{k}$} & SLG & BLG \\
\hline & & & $\mathrm{I}_{\mathrm{hk}} \times 10^{6}$ & $\mathrm{I}_{\mathrm{hk}} \times 10^{6}$ \\
\hline & 1 & 0 & & \\
\hline$\{10.0\}_{\mathrm{A}}$ & 0 & -1 & 70.6 & 62.3 \\
\hline & -1 & 0 & & \\
\hline$\{-10.0\}_{\mathrm{B}}$ & 0 & 1 & 70.0 & 74.6 \\
\hline & 1 & -1 & & \\
\hline & 1 & 1 & & \\
\hline$\{11.0\}_{\mathrm{A}}$ & 1 & -2 & 57.8 & 230.2 \\
\hline & -2 & 1. & & \\
\hline & -1 & -1 & & \\
\hline$\{-1-1.0\}_{B}$ & -1 & 2 & 57.8 & 230.2 \\
\hline & 2 & -1 & & \\
\hline
\end{tabular}

Table 1. The calculated dynamical electron diffraction intensity of $\{$ hk. 0$\}$ reflections $\mathrm{I}_{\mathrm{hk}}=\left|\phi_{\mathrm{hk}}\right|^{2}$ for single layer graphene (SLG with thickness $\mathrm{t}=0.354$ $\mathrm{nm}$ ) and bilayer graphene (BLG: $\mathrm{t}=0.671 \mathrm{~nm}$ ) in [0001] orientation with 200 $\mathrm{keV}$ incident electron. Please note that the violation of Friedel's law in $\{10.0\}$ reflections for BLG.

Table 2. The measured intensity of diffraction spots from the diffraction pattern of Fig. 3(A) in [4]. It is reported in [4] that the pattern was taken by a 200 FE-TEM $(200 \mathrm{kV})$ operated with STEM parallel beam mode. This figure shows the seven sets of diffraction patterns obtained from the sample of overlapped and folded sheets of SLG and BLG. Among the seven sets the four sets of the patterns were chosen for the intensity measurements as listed

\begin{tabular}{|c|c|c|c|c|c|c|c|c|}
\hline & \multicolumn{2}{|c|}{ No. 1} & \multicolumn{2}{|c|}{ No. 2} & \multicolumn{2}{|c|}{ No. 3} & \multicolumn{2}{|c|}{ No. 4} \\
\hline h $\mathrm{k}$ & $\mathrm{I}_{\mathrm{hk}}$ & $<\mathrm{I}_{\mathrm{hk}}>$ & $\mathrm{I}_{\mathrm{hk}}$ & $<\mathrm{I}_{\mathrm{hk}}>$ & $\mathrm{I}_{\mathrm{hk}}$ & $<\mathrm{I}_{\mathrm{hk}}>$ & $\mathrm{I}_{\mathrm{hk}}$ & $<\mathrm{I}_{\mathrm{hk}}>$ \\
\hline $\begin{array}{ll}1 & 0\end{array}$ & 115 & & 88 & & 151 & & 182 & \\
\hline $\begin{array}{ll}0 & -1\end{array}$ & 56 & $85 \pm 30$ & 92 & $99 \pm 17$ & 107 & $149 \pm 42$ & 173 & $170 \pm 16$ \\
\hline $\begin{array}{ll}-1 & 1\end{array}$ & 84 & & 116 & & 188 & & 154 & \\
\hline-10 & 73 & & 83 & & 159 & & 179 & \\
\hline $\begin{array}{ll}0 & 1\end{array}$ & 84 & $86 \pm 16$ & 101 & $105 \pm 25$ & 127 & $158 \pm 31$ & 157 & $155 \pm 26$ \\
\hline $\begin{array}{ll}1 & -1 \\
\end{array}$ & 102 & & 130 & & 189 & & 129 & \\
\hline $\begin{array}{ll}1 & 1\end{array}$ & 62 & & 67 & & 200 & & 247 & \\
\hline $\begin{array}{ll}1 & -2\end{array}$ & 59 & $64 \pm 6$ & 57 & $64 \pm 7$ & 180 & $192 \pm 12$ & 239 & $242 \pm 5$ \\
\hline $\begin{array}{r}-2 \\
-1\end{array}$ & 70 & & 69 & & 197 & & 240 & \\
\hline$-1-1$ & 27 & & 89 & & 223 & & 237 & \\
\hline $\begin{array}{ll}-1 & 2\end{array}$ & 32 & $34 \pm 8$ & 74 & $78 \pm 11$ & 186 & $193 \pm 30$ & 238 & $233 \pm 10$ \\
\hline $\begin{array}{ll}2 & -1 \\
\end{array}$ & 42 & & 71 & & 170 & & 223 & \\
\hline
\end{tabular}
in the table. The diffraction spots with circles shown in the pattern in [4] is No. 2 set; No.1 set is with the $5^{\text {th }}$ spot from the top spot (one of $\{11.0\}$ ) in the clockwise rotation; No. 3 set is with the $4^{\text {th }}$ spot from the top; No. 4 set is with the $1^{\text {st }}$ spot from the top. No. 1 and No. 2 reflections seem to be for SLG; No. 3 and No. 4 to be for BLG, as comparing the intensity ratio of $\{10.0\}$ and $\{11.0\}$ reflections in Table 1 and those in Table 2 . 